\section{A equidade na cobertura da Estratégia Saúde da Família em Minas Gerais, Brasil}

\author{
Equity in coverage by the Family Health Strategy \\ in Minas Gerais State, Brazil
}

\section{La equidad en la cobertura de la Estrategia de Salud Familiar en Minas Gerais, Brasil}

\begin{abstract}
The Family Health Strategy (FHS) plays an important role in prevention and in monitoring families in the Brazilian Unified National Health System. This study aims to analyze equity in the coverage of these services in the urban areas of Minas Gerais State, Brazil. The research is unprecedented and analyzes several markers for four target groups: women, pregnant women, children, and the elderly. The study is representative of the various health macro-regions. In 2012, 6,797 households were surveyed, with 5,820 women, 1,758 children, and 3,629 elderly. To analyze equity, FHS coverage rates were calculated according to family income, and concentration indices and curves were estimated. The results show that the FHS is an equitable policy. The indicators show that poorer households have higher visitation rates under the FHS. Coverage of the eligible population is quite high: $88 \%$ of households received at least one visit from FHS professionals in the previous 12 months, resulting in a concentration index near zero.
\end{abstract}

Family Health Strategy; Primary Health Care; Equity in Health

\author{
Mônica Viegas Andrade 1 \\ Kenya Noronha 1 \\ Allan Claudius Queiroz Barbosa 1 \\ Thiago Augusto Hernandes Rocha 1 \\ Núbia Cristina da Silva 1 \\ Júlia Almeida Calazans 1 \\ Michelle Nepomuceno Souza 1 \\ Lucas Resende de Carvalho 1 \\ Aline Souza ${ }^{1}$
}

\section{Resumo}

A Estratégia Saúde da Família (ESF) tem papel relevante na prevenção e no acompanhamento das famílias no Sistema Único de Saúde. O presente estudo tem como objetivo analisar a equidade na cobertura desses serviços ofertados na área urbana de Minas Gerais, Brasil. A pesquisa analisa diversos marcadores considerando quatro grupos-alvo: mulheres, gestantes, crianças e idosos, sendo representativa por macrorregião de saúde. Foram investigados em 2012, 6.797 domicílios, sendo entrevistados 5.820 mulheres, 1.758 crianças e 3.629 idosos. Para analisar a equidade, foram construídas taxas de cobertura da ESF por classe de riqueza e estimados índices e curvas de concentração. Os resultados revelam que a ESF é uma política equitativa. Os indicadores mostram que os domicílios mais pobres apresentam maiores taxas de visitação da ESF. Considerando a população residente nas áreas adscritas às equipes de saúde, o nível de cobertura é bastante elevado: $88 \%$ da população investigada receberam pelo menos uma visita dos profissionais da ESF nos últimos 12 meses, o que resulta em índices de concentração perto de zero.

Estratégia Saúde da Família; Atenção Primária à Saúde; Equidade em Saúde 


\section{Introdução}

As últimas décadas no Brasil vêm sendo marcadas por importantes mudanças sociais. Entre as principais políticas sociais cabe mencionar os programas de transferência de renda, a expansão da cobertura escolar e a consolidação do Sistema Único de Saúde (SUS) 1,2. Com a criação do SUS, em 1988, o Estado brasileiro assumiu a responsabilidade da oferta e financiamento de todos os serviços de saúde. Desde então, diversas políticas têm sido implementadas buscando garantir uma oferta mais eficiente e mais equitativa ${ }^{3}$. Os resultados dessas políticas podem ser observados nas melhoras no acesso aos serviços de saúde, com destaque para a atenção primária e emergência, incluindo cuidado pré-natal, cobertura vacinal e acesso a medicamentos 2. Estudos recentes mostram aumentos nas taxas de utilização dos serviços de saúde nos últimos dez anos, especialmente entre as camadas de renda mais baixa, o que vem contribuindo para reduzir iniquidades na utilização desses serviços 4,5.

Parte desses avanços pode ser explicada pela ampliação da atenção primária que vem ocorrendo em todo o país. Em meados da década de 1990, o Ministério da Saúde deu início a um processo de mudança no SUS, passando de um modelo assistencial tradicional centrado na doença e no cuidado médico individualizado para um modelo que prioriza a atenção primária. No modelo anterior o foco é no cuidado curativo e hospitalocêntrico, o novo modelo é focado na promoção, prevenção e acompanhamento da população usuária. A criação do Programa Saúde da Família (PSF), em 1994, foi um reflexo dessa mudança 6,7,8. Em 2006, foi aprovada a primeira Política Nacional de Atenção Básica (PNAB) 8, que transformou o PSF em uma estratégia de abrangência nacional denominada a partir de então Estratégia Saúde da Família (ESF). Essa política foi reeditada em 2011 em função da necessidade de se discutir, principalmente, a questão da qualidade na oferta de serviços de saúde 9,10. Atualmente, a ESF está configurada como a principal iniciativa de atenção primária desenvolvida no âmbito do SUS, tendo papel relevante na prevenção e no acompanhamento das famílias. $\mathrm{O}$ atendimento da população é orientado pelos seguintes princípios ordenadores: primeiro contato, integralidade, coordenação, longitudinalidade, orientação comunitária, foco na família e competência cultural 10 .

Ainda que diversos avanços tenham sido alcançados desde a criação do SUS e da ESF, as desigualdades regionais e socioeconômicas no acesso aos serviços de saúde no país ainda são marcantes, sendo mais acentuadas para os cui- dados preventivos 11,12,13,14,15,16,17,18. Nas últimas décadas, essas desigualdades têm sofrido expressiva redução, que pode ser atribuída tanto à ampliação do acesso aos serviços de saúde entre a população SUS dependente, promovida pela ESF $19,20,21$, quanto pelas melhoras no padrão de vida da população.

Em 2012, foi realizada uma pesquisa visando a analisar os serviços ofertados pela ESF no Estado de Minas Gerais. A pesquisa investigou os principais marcadores da atenção primária para crianças, gestantes, idosos e mulheres em idade reprodutiva. Esse é um primeiro estudo desenhado para avaliar o papel da ESF na redução das desigualdades na utilização dos serviços de saúde. O objetivo deste artigo é apresentar essa pesquisa ressaltando os resultados atinentes à cobertura da ESF, bem como da desigualdade social nesta cobertura, em Minas Gerais e por região ampliada de saúde.

\section{Procedimentos metodológicos}

\section{Minas Gerais e a saúde da família}

Minas Gerais possui cerca de 20 milhões de habitantes distribuídos em 853 municípios. O estado é caracterizado por forte heterogeneidade socioeconômica e demográfica. Em 2010, o PIB per capita anual estava em torno de $\mathrm{R} \$ 10.700,00$, sendo R\$26.144,00 na macrorregião do Triângulo do Sul e R\$ 6.003,00 na do Jequitinhonha. Essa disparidade também é observada para a escolaridade e indicadores epidemiológicos: em 2010, a taxa de analfabetismo variava de $4,7 \%$ no Centro a $22,5 \%$ no Nordeste; e a taxa de mortalidade infantil variava de 10,3 no Município de Passos a 27,8 em Santa Helena de Minas. Nas duas últimas décadas, o estado passou por mudanças demográficas e socioeconômicas importantes, caracterizadas, sobretudo, pela redução da desigualdade e da pobreza. Entre os anos de 1990 e 2009, o coeficiente de Gini, utilizado para medir a desigualdade de renda, reduziu de 0,63 para 0,54 , representando uma queda de $19 \%$, superior à média nacional de $12 \%$. Ademais, a taxa de pobreza em Minas Gerais variou de 38,27\% para $12,05 \%$, configurando uma redução de $70 \%$ (Instituto de Pesquisa Econômica Aplicada. http://www.ipeadata.gov.br, acessado em 30/ Nov/2011). Contudo, as desigualdades socioeconômicas entre as regiões ainda são expressivas. Minas Gerais possui 4.253 equipes de atenção primária, ou seja, $14 \%$ do total do país. A atenção primária é fortalecida pelo Projeto Saúde em Casa e pela implantação das redes de assistência à saúde, com foco na rede de hipertensão e dia- 
betes (HIPERDIA), atenção à saúde da mulher e crianças (Rede Viva Vida) e a rede de atenção às pessoas idosas (Rede Mais Vida).

\section{Desenho do estudo}

Foram realizados inquéritos domiciliares na área urbana, com amostra representativa para quatro grupos-alvo da ESF: crianças menores de cinco anos, idosos (60 anos e mais), gestantes e mulheres entre 25 e 59 anos. Foram consideradas gestantes as mulheres que tinham filhos menores de cinco anos vivos presentes no domicílio. Essa definição permitiu obter uma amostra maior de mulheres com informação sobre todo o período gestacional, muito embora possa resultar em um viés de memória. A área rural foi excluída do estudo devido às dificuldades associadas à logística de campo que comprometeriam o orçamento disponível para a realização desta pesquisa. O alcance dos resultados diz respeito à implementação da ESF na área urbana de Minas Gerais, onde $85 \%$ da população residem. O trabalho foi aprovado pelo Comitê de Ética em Pesquisa da Universidade Federal de Minas Gerais (COEP/UFMG; parecer 04200203000-10). O instrumento de coleta dos dados foi organizado em sete seções que se referem à identificação e caracterização sociodemográfica do domicílio e características de saúde dos quatro grupos-alvo.

\section{- Amostragem das equipes investigadas}

O desenho da amostra foi probabilístico, no qual a unidade primária de seleção era a equipe da ESF e a unidade secundária, o domicílio. A amostra foi uniformemente distribuída entre as macrorregiões de saúde. O desenho amostral foi estratificado por região, com alocação igual de 16 equipes da ESF em cada macrorregião de saúde, respeitando a proporção dos grupos-alvo na população em Minas Gerais. Com essa alocação igualitária de número de equipes por região, os erros de estimação nos estratos tendem a ficar homogêneos.

Em cada macrorregião, efetuou-se a seleção aleatória sistemática dos municípios de acordo com o Índice de Desenvolvimento Humano (IDH), garantindo maior variabilidade socioeconômica entre os municípios selecionados. A probabilidade de seleção do município foi proporcional ao número de equipes de saúde da família. Foram selecionados 173 municípios e em seguida procedeu-se ao sorteio de equipes de saúde naqueles que em 2011 tinham mais de uma equipe. No caso de a equipe sorteada pertencer à área rural, foi realizada a substituição.
O tamanho amostral foi definido de modo a garantir a representatividade por macrorregião de saúde e grupo-alvo, considerando a probabilidade de ocorrência de cada evento de interesse associado aos grupos-alvo. Para as crianças, o evento de interesse foi a posse do cartão de vacina; para idosos, considerou-se a utilização de consultas médicas e para mulheres, a cobertura de exame preventivo.

\section{- Amostragem probabilística de domicílios}

Definidos os municípios e as equipes, procedeu-se à seleção aleatória dos domicílios. A população de interesse compreende os indivíduos cobertos pela ESF residentes nas áreas adstritas a cada equipe. Uma proposta natural seria utilizar os usuários cadastrados na ESF. Nesse caso, seriam amostrados somente os indivíduos que utilizam os serviços oferecidos pela ESF, levando ao viés de seleção. Outra opção seria utilizar o recorte geográfico das áreas de abrangência das equipes. Essa informação não está disponível para todas as equipes do Estado de Minas Gerais. Optou-se nesta amostragem pela construção do que se denominou de "área de abrangência potencial”. Para construir essa área considerou-se o protocolo do Ministério da Saúde que define que cada equipe é responsável por acompanhar em média 3.450 indivíduos. Supondo 4 moradores por domicílio, a área de abrangência potencial seria composta por, aproximadamente, 850 domicílios localizados em setores censitários geograficamente próximos à Unidade Básica de Saúde (UBS). Procedeu-se então ao georreferenciamento das UBS e subsequentemente foram selecionados os 850 domicílios situados nos setores censitários contíguos registrados no Cadastro Nacional de Endereços para Fins Estatísticos (CNEFE). Na área de abrangência potencial, os domicílios foram sorteados aleatoriamente. Para lidar com a possibilidade de perdas devido ao desenho probabilístico, o número de domicílios sorteados foi sobreamostrado em $50 \%$. Nos municípios onde a cobertura da ESF era superior a 95\% da população a área de abrangência potencial considerada foi todo o município.

Foram sorteados 10.311 domicílios, aproximadamente 800 para cada macrorregião. Desse total, 3.247 não possuíam algum morador do grupo-alvo ou o endereço sorteado era não existente. Dentre os 7.064 domicílios elegíveis, apenas em 267 houve recusa em participar da pesquisa, totalizando 6.797 domicílios visitados. Para cada domicílio visitado foram incluídos na pesquisa todos os moradores que pertenciam a algum dos quatro grupos-alvo investigados. Em $50 \%$ dos domicílios só foi entrevistado um mora- 
dor. Para os domicílios onde foram entrevistados os grupos de crianças, gestantes e mulheres, mais de $80 \%$ só apresentavam um morador referente ao grupo-alvo. Dos domicílios com idosos, $66 \%$ tinham apenas um morador desse grupo-alvo.

\section{Variáveis utilizadas}

A equidade na cobertura da ESF foi avaliada considerando como unidade de análise o domicílio. A cobertura foi analisada para o universo dos domicílios e posteriormente desagregando segundo a presença de pelo menos um morador pertencente aos seguintes grupos-alvo: mulheres entre 25 e 59 anos, crianças menores de cinco anos e idosos. Além disso, a cobertura da ESF nos grupos-alvo foi também analisada considerando a existência de doença crônica. Para mulheres e idosos, foram selecionados os domicílios com a presença de pelo menos um morador do grupo-alvo correspondente, portador de diabetes e hipertensão, as duas principais condições crônicas acompanhadas pela ESF. Para as crianças, foi analisada a cobertura da ESF entre domicílios com crianças portadoras de pelo menos uma das seguintes condições crônicas: doenças respiratórias, anemia, desnutrição e diarreia persistente, que são consideradas as mais prevalentes na infância de acordo com o protocolo de atenção à saúde da criança da Secretaria Estadual de Saúde de Minas Gerais. A existência de cada uma dessas doenças foi avaliada com base em medidas autorreportadas. Perguntou-se aos indivíduos se algum médico ou profissional de saúde disse se ele/ela tem ou teve cada uma das doenças crônicas investigadas.

$\mathrm{O}$ indivíduo foi classificado como coberto pela ESF se o domicílio em que residia tivesse recebido pelo menos uma visita nos últimos 12 meses que antecederam a data da entrevista. Foi considerada a visita de qualquer profissional de saúde da ESF, incluindo os agentes comunitários de saúde (ACS), auxiliares e técnicos de enfermagem, enfermeiro, médicos e outros profissionais. Neste artigo, a cobertura da ESF para as gestantes não é analisada, pois foi aferida a visita de um profissional da ESF somente no último ano e a definição de gestante considera como período de referência os últimos cinco anos.

$\mathrm{O}$ indicador socioeconômico foi gerado com base no Critério de Classificação Econômica Brasil desenvolvido pela Associação Brasileira de Empresas de Pesquisa (http://www.abep.org/ new). Nessa metodologia são atribuídos pontos à posse de bens específicos (rádio, máquina de lavar, televisão em cores, vídeo/DVD, geladeira, freezer e automóvel), bem como instalações (número de banheiros), número de emprega- dos mensalistas e grau de instrução do chefe do domicílio. No presente estudo, a informação sobre escolaridade refere-se ao grau máximo de estudos encontrado no domicílio. Essa escolha decorre da dificuldade em se identificar o chefe nos arranjos domiciliares menos tradicionais. $\mathrm{O}$ somatório desses pontos gera um escore de riqueza, que permite a classificação dos domicílios em sete classes econômicas (A1, A2, B1, B2, C, D e E). Tendo em vista o pequeno número de observações, os domicílios foram classificados em três classes sociais: A-B, C e D-E. A análise foi realizada para o estado e para as 13 macrorregiões de saúde definidas em 201122.

\section{Índice de concentração}

O índice de concentração (IC) fornece uma medida sintética da extensão da desigualdade social em saúde. O IC mede a relação entre a proporção acumulada da população, ordenada de forma crescente pelo nível socioeconômico, contra a proporção acumulada de indivíduos de acordo com a utilização ou acesso a serviços de saúde 23 . Os valores do IC variam entre -1 e 1 . Um valor igual a zero indica ausência de desigualdade social e o valor igual a 1 ou -1 indica que somente os indivíduos mais ricos ou mais pobres, respectivamente, apresentam o atributo de saúde analisado. Portanto, IC positivo indica que a cobertura da ESF está concentrada entre os indivíduos mais ricos e IC negativo indica concentração entre os mais pobres.

A estimação do IC é realizada por meio de uma regressão de mínimos quadrados ordinários (MQO) conforme proposto por O'Donnell et al. 23. Nessa abordagem, o IC é obtido usando-se a covariância entre a posição socioeconômica e a cobertura da ESF. No modelo de MQO, a variável dependente é uma transformação da variável de cobertura da ESF. Essa transformação considera a distância de cada indivíduo em relação à cobertura média ponderada pela variância do score de riqueza. A variável independente de interesse é a posição do indivíduo na distribuição acumulada do score de riqueza. O coeficiente estimado refere-se ao IC e o nível de significância é avaliado com base no teste $t$ de Student corrigindo pela variabilidade amostral. Além da variável independente de interesse, variáveis binárias para cada região ampliada de saúde de Minas Gerais foram incluídas no modelo de regressão para a estimação do IC.

\section{Curva de concentração}

A representação gráfica do IC é a curva de concentração (CC). Similarmente à curva de Lorenz, 
a área delimitada pela curva e pela diagonal mede o IC. A diagonal representa perfeita igualdade na distribuição dos serviços de saúde entre os grupos socioeconômicos. Se a CC estiver posicionada abaixo da diagonal, o IC é positivo. Se a CC estiver acima da diagonal, o IC é negativo. Quando a CC coincide com a diagonal, o IC é zero. A CC pode cruzar a diagonal. Quando isso ocorre, a área acima da diagonal pode compensar a área abaixo da diagonal, resultando em um índice de concentração que subestima a iniquidade. Nesse caso, é importante analisar o comportamento do IC conjuntamente ao gráfico da CC. Para testar a dominância estatística das curvas de concentração em relação à linha de equidade, foi realizado o teste que compara estas duas distribuições acumuladas com base no intersection union principle (IUP) 23. A hipótese nula testada é a de que não há dominância entre a CC e a linha de equidade. Para que a hipótese H0 seja rejeitada, a proporção acumulada da população coberta pela ESF deve ser estatisticamente diferente da proporção acumulada da população em todos os pontos de riqueza. No caso de haver diferença estatisticamente significativa em pelo menos um ponto da distribuição de riqueza nas duas direções, conclui-se que a curva de concentração cruza a diagonal. Para a realização do teste de dominância, foram definidos 19 quantis igualmente espaçados ao longo de toda a distribuição. $\mathrm{O}$ critério IUP é considerado rigoroso por analisar todos os pontos da distribuição. O teste tem a vantagem de reduzir a chance de se rejeitar a hipótese nula erroneamente, mas aumenta a chance de aceitar a hipótese nula quando esta é falsa.

\section{Resultados}

A pesquisa entrevistou 6.797 domicílios localizados em áreas de abrangência potencial da ESF em todo o estado. Foram entrevistados $5.820 \mathrm{mu}$ lheres, 1.758 crianças e 3.629 idosos. Observouse que $88 \%$ dos domicílios investigados declararam ter recebido pelo menos uma visita de algum profissional da ESF nos 12 meses anteriores à data da entrevista, sendo que cada um dos domicílios cobertos recebeu em média 10,40 visitas durante o período. Essa elevada cobertura não é uniforme entre as regiões de saúde. A Tabela 1 mostra a presença de disparidade regional da cobertura da ESF em Minas Gerais. Nas regiões de saúde mais pobres a taxa de visitação é em torno de $90 \%$, com destaque para a região Norte, onde $93,16 \%$ dos domicílios foram visitados. Por outro lado, na macrorregião Centro somente $77,86 \%$ dos domicílios receberam alguma visita, o que mostra a menor inserção da Estratégia em áreas mais desenvolvidas.

Tabela 1

Distribuição dos domicílios entrevistados por visitação da Estratégia Saúde da Família, segundo macrorregiões de saúde de Minas Gerais, Brasil, 2012

\begin{tabular}{|c|c|c|c|c|}
\hline \multirow[t]{2}{*}{ Macrorregiões } & \multicolumn{2}{|c|}{ Não visitados } & \multicolumn{2}{|c|}{ Visitados } \\
\hline & $\mathrm{n}$ & $\%$ & $\mathbf{n}$ & $\%$ \\
\hline Centro & 116 & 22,14 & 408 & 77,86 \\
\hline Centro-sul & 68 & 10,64 & 571 & 89,36 \\
\hline Jequitinhonha & 61 & 9,78 & 563 & 90,22 * \\
\hline Leste & 52 & 13,30 & 339 & 86,70 \\
\hline Leste do Sul & 54 & 11,04 & 435 & 88,96 ** \\
\hline Nordeste & 60 & 14,78 & 346 & 85,22 \\
\hline Noroeste & 60 & 9,00 & 607 & 91,00 * \\
\hline Norte & 37 & 6,84 & 504 & 93,16 * \\
\hline Oeste & 48 & 9,07 & 481 & 90,93 \\
\hline Sudeste & 47 & 12,67 & 324 & 87,33 \\
\hline Sul & 65 & 12,87 & 440 & 87,13 \\
\hline Triângulo do Norte & 100 & 17,45 & 473 & 82,55 \\
\hline Triângulo do Sul & 79 & 14,79 & 455 & 85,21 \\
\hline Total & 847 & 12,47 & 5.946 & 87,53 \\
\hline
\end{tabular}

Fonte: Pesquisa Equidade na Saúde: Estratégia Saúde da Família em Minas Gerais (2012).

* Diferença de cobertura em relação à média estatisticamente significativa a 1\%;

** Diferença de cobertura em relação à média estatisticamente significativa a $5 \%$. 
Domicílios das classes de riqueza menos favorecidas são mais cobertos pela ESF do que os domicílios das classes mais favorecidas (Tabela 2). Enquanto $75 \%$ dos domicílios das classes A-B declararam ter recebido pelo menos uma visita dos profissionais da ESF nos 12 meses anteriores à pesquisa, entre as classes D-E este percentual foi mais elevado, igual a $88 \%$. Essas diferenças são estatisticamente significativas em relação à média total do estado.

A análise do IC corrobora esses resultados. Como pode ser observado na Tabela 2, o IC é negativo $(-0,0266)$ e estatisticamente significativo, indicando uma leve desigualdade em favor dos mais pobres. O teste de dominância mostra que a CC (Figura 1) cruza a linha de equidade. A análise visual do gráfico mostra que esse cruzamento ocorre nos primeiros percentis da distribuição de riqueza, sugerindo que a desigualdade na cobertura da ESF a favor dos mais pobres ocorre somente em uma parte da distribuição, mais evidente a partir do quarto decil de riqueza.

A análise por região evidencia o Centro como aquela que possui a menor cobertura da ESF e as maiores disparidades favoráveis aos mais pobres. Enquanto 60,08\% dos domicílios das classes A-B receberam pelo menos uma visita dos profissio- nais no período de análise, este porcentual nos domicílios das classes D-E é igual a 84,6\% (Tabela 2). Além da região Centro, o Nordeste, Noroeste, Sudeste e Sul também apresentam expressivas diferenças socioeconômicas, com a cobertura da Estratégia mais focalizada nos domicílios mais pobres. Nessas regiões, o IC da cobertura é negativo e estatisticamente significativo, indicando desigualdade em favor das classes mais pobres. As curvas de concentração para as macrorregiões cujos IC são estatisticamente significativos podem ser visualizadas na Figura 1. A análise visual dos gráficos evidencia que para todas essas regiões a CC da cobertura do ESF está acima ou sobreposta à linha de $45^{\circ}$. O teste de dominância reforça esse resultado reportando cruzamento das curvas ou não dominância (Tabela 2). A opção pelo critério IUP para análise de dominância entre as curvas implica resultados mais conservadores na medida em que o teste só rejeita a hipótese nula no caso de haver diferença estatisticamente significativa em todos os pontos da distribuição. Dessa forma, o teste de dominância indica equidade na cobertura da ESF embora o IC reporte que essa distribuição é levemente favorável aos mais pobres. Essa discrepância entre as duas estatísticas se deve ao fato de o teste de

Tabela 2

Taxa de visitação dos domicílios pelos profissionais de saúde da Estratégia Saúde da Família nos 12 meses anteriores à data da entrevista, por classe de riqueza e índice de concentração (IC), segundo macrorregiões de saúde de Minas Gerais, Brasil, 2012.

\begin{tabular}{|c|c|c|c|c|c|c|}
\hline Macrorregiões & Classes A-B & Classe C & Classes D-E & IC & $\begin{array}{l}\text { Valor de } p \\
\text { do IC }\end{array}$ & $\begin{array}{l}\text { Teste de dominância } \\
\text { (IUP) }\end{array}$ \\
\hline Centro & 60,08 * & 81,82 & 84,59 ** & $-0,062$ & 0,000 & Cruzamento \\
\hline Centro-sul & 89,33 & 89,75 & 91,78 & $-0,003$ & 0,720 & Não dominância \\
\hline Jequitinhonha & 87,34 & 93,72 & 89,83 & $-0,001$ & 0,932 & Não dominância \\
\hline Leste & 81,79 & 84,66 & 84,01 & 0,002 & 0,897 & Cruzamento \\
\hline Leste do Sul & 86,54 & 90,85 & 89,88 & $-0,005$ & 0,575 & Não dominância \\
\hline Nordeste & 73,18 & 82,01 & 91,38 & $-0,029$ & 0,017 & Cruzamento \\
\hline Noroeste & 84,12 & 90,33 & 93,62 & $-0,024$ & 0,002 & Cruzamento \\
\hline Norte & 89,74 & 92,77 & 96,04 & $-0,010$ & 0,128 & Cruzamento \\
\hline Oeste & 90,32 & 90,90 & 88,41 & 0,004 & 0,651 & Não dominância \\
\hline Sudeste & 77,71 & 85,00 & 92,19 & $-0,031$ & 0,013 & Cruzamento \\
\hline Sul & $76,60 * \star$ & 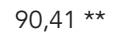 & 88,99 & $-0,036$ & 0,001 & Não dominância \\
\hline Triângulo do Norte & 90,56 & 77,85 & 89,33 ** & $-0,010$ & 0,354 & Cruzamento \\
\hline Triângulo do Sul & 78,49 & 86,51 & 85,47 & $-0,001$ & 0,889 & Não dominância \\
\hline Total & 75,14 * & 85,55 * & 88,17 * & $-0,027$ & 0,000 & Cruzamento \\
\hline
\end{tabular}

IUP: intersection union principle.

Fonte: Pesquisa Equidade na Saúde: Estratégia Saúde da Família em Minas Gerais (2012).

* Diferença de médias em relação à media geral da região significativa a $1 \%$;

** Diferença de médias em relação à media geral da região significativa a $5 \%$. 
Curvas de concentração para visitas dos profissionais de saúde da Estratégia Saúde da Família nos 12 meses anteriores a data da entrevista, por macrorregião de saúde. Minas Gerais, Brasil, 2012.

1a) Total

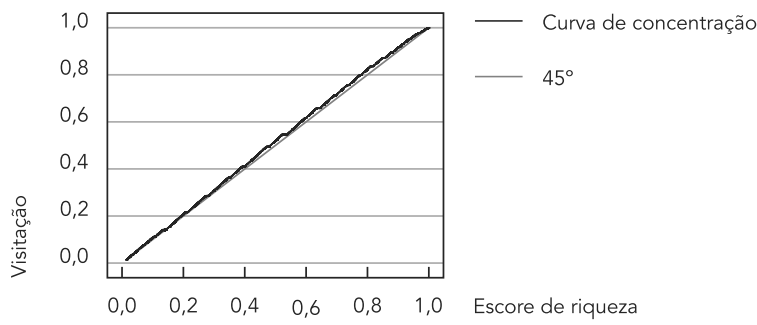

1c) Nordeste

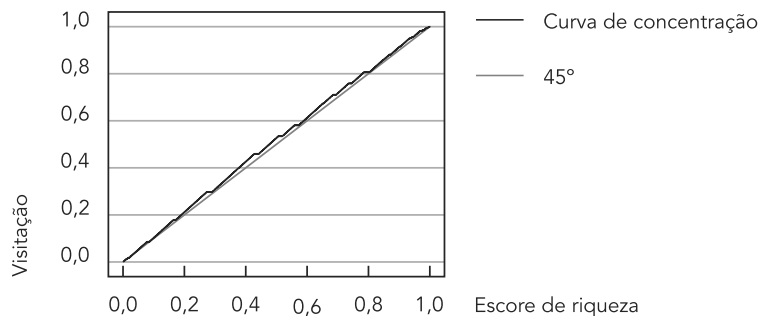

1e) Sudeste

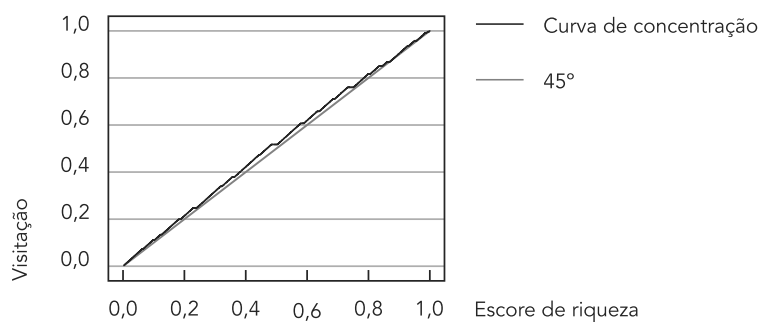

1b) Centro

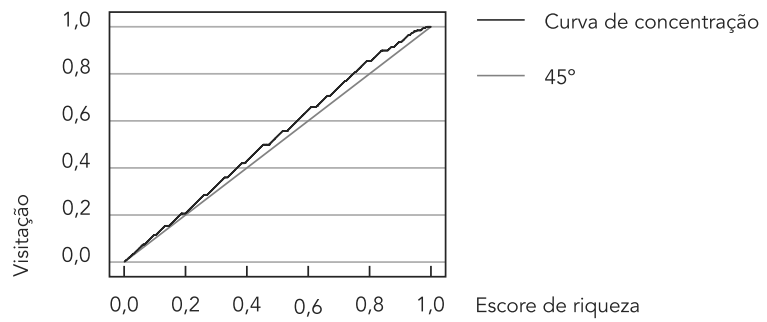

1d) Noroeste

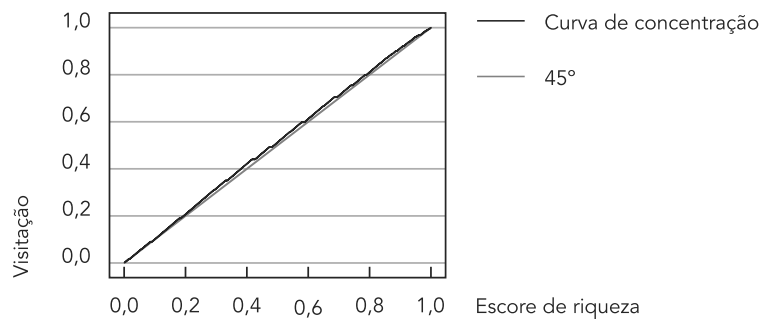

1f) Sul

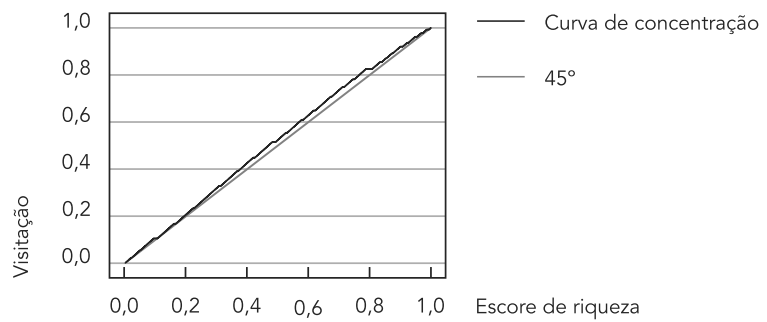

Fonte: Pesquisa Equidade na Saúde: Estratégia Saúde da Família em Minas Gerais (2012).

dominância considerar toda a distribuição, assim, pontos de tangência da CC com a diagonal induzem o resultado de não dominância ou de cruzamento. As macrorregiões Centro-sul, Jequitinhonha, Leste, Leste do Sul, Norte, Oeste, Triângulo do Norte e Triângulo do Sul não apresentam IC estatisticamente significativo. Nessas localidades, os resultados sugerem equidade na cobertura da ESF

Esse padrão de desigualdade socioeconômica favorável aos mais pobres é observado para todos os grupos-alvo investigados (Tabela 3). Em média, 83\% dos domicílios com pelo menos uma mulher entre 25 e 59 anos receberam pelo menos uma visita, observando uma diferença de quase 10 pontos percentuais na cobertura entre as classes A-B e D-E. Essa desigualdade é ainda mais acentuada no caso das mulheres com diabetes e hipertensão. Sessenta e sete por cento dos domicílios com mulheres com diabetes das classes A-B receberam visita da ESF, entre as classes D-E este percentual é igual a $93 \%$. 
Entre os domicílios com a presença de crianças menores de 5 anos, cerca de $82 \%$ receberam pelo menos uma visita ao longo do período investigado. Essa cobertura varia de acordo com a classe econômica, sendo que as diferenças mais acentuadas são encontradas nos domicílios que têm crianças com doenças crônicas: $70 \%$ nos domicílios das classes A-B contra $88 \%$ nos das classes D-E.

Domicílios com idosos são aqueles que apresentam as maiores taxas de visitação (87\%), sendo que com a existência de indivíduos com diabetes e hipertensão este percentual é mais elevado. Mesmo tendo um nível de cobertura elevada ainda persistem diferenciais socioeconômicos de cobertura favoráveis aos mais pobres. Noventa e dois por cento dos domicílios das classes $\mathrm{D}$-E receberam alguma visita dos profissionais da ESF, este percentual é de $71 \%$ nas classes A-B.

Os IC foram estimados controlando para a macrorregião de residência. Para todos os grupos populacionais investigados, o IC é negativo e estatisticamente significativo, reforçando os resultados da análise descritiva. O resultado do IC indica uma concentração da cobertura em favor dos domicílios mais pobres (Tabela 3). A Figura 2 apresenta as CC por grupo-alvo. O teste de dominância mostrou pelo critério IUP que nenhuma das curvas domina a linha de equidade.

\section{Discussão e considerações finais}

Este artigo analisa os diferenciais socioeconômicos da cobertura da ESF em Minas Gerais. Os resultados revelam que a ESF é uma política equi- tativa, no sentido que favorece aos mais pobres e reduz as diferenças socioeconômicas ao acesso a serviços de saúde atinentes à atenção primária no estado. Considerando a população residente nas áreas adscritas às equipes de saúde, o nível de cobertura é bastante elevado: $88 \%$ da população investigada receberam pelo menos uma visita dos profissionais da ESF nos últimos 12 meses. Essa elevada cobertura se traduz em uma menor desigualdade social no acesso a esses serviços. Embora os domicílios mais pobres sejam, proporcionalmente, mais cobertos que os mais ricos por apresentarem maiores taxas de visitação, os IC são próximos a zero. Considerando a desagregação por macrorregião de saúde, apenas para o Centro, Nordeste, Noroeste, Sudeste e Sul, o IC da cobertura é estatisticamente significativo e indica uma pequena desigualdade em favor das classes mais pobres.

A menor cobertura da ESF foi observada nas áreas mais desenvolvidas e nas classes econômicas mais elevadas. Tendo em vista a dificuldade de implementar a ESF nos grandes centros urbanos, o Ministério da Saúde vem concentrando esforços, desde o ano 2000, para induzir a expansão, com qualidade, da ESF em regiões de aglomerados urbanos. Segundo o Ministério da Saúde (Sistemas de Informação da Atenção Básica. http:// dab.saude.gov.br/dab/historico_cobertura_sf/ historico_cobertura_sf_relatorio.php, acessado em 29/Ago/2013), a cobertura populacional da ESF no Brasil vem crescendo ao longo dos últimos anos, passando de 31,88\% em novembro de 2002 para $61,6 \%$ em setembro de 2014. Em Minas Gerais, a cobertura cresceu de $39,65 \%$ para $77,6 \%$ no mesmo período, sendo superior à média nacional

Tabela 3

Taxa de visitação dos domicílios pelos profissionais de saúde da Estratégia Saúde da Família nos 12 meses anteriores à data da entrevista, por classe de riqueza e índice de concentração (IC), segundo a presença de grupo-alvo no domicílio. Minas Gerais, Brasil, 2012.

\begin{tabular}{|c|c|c|c|c|c|c|}
\hline Grupo-alvo & Classes A-B & Classe C & Classes D-E & $\begin{array}{l}\text { IC com controle } \\
\text { por região }\end{array}$ & $\begin{array}{l}\text { Valor de } p \\
\text { do IC }\end{array}$ & Teste de dominância (IUP) \\
\hline Mulheres & $76,69 *$ & $84,50 * \star$ & 86,42 * & $-0,024$ & 0,000 & Cruzamento \\
\hline Mulheres com diabetes & 66,62 * & 89,95 & 92,83 * & $-0,070$ & 0,000 & Cruzamento \\
\hline Mulheres com hipertensão & 75,53 * & 89,93 * & 86,62 & $-0,026$ & 0,000 & Cruzamento \\
\hline Crianças & $76,32 \star \star$ & 83,75 & 85,63 & $-0,020$ & 0,013 & Não dominância \\
\hline Crianças com doença crônica & 69,74 ** & 85,49 & 88,48 & $-0,057$ & 0,002 & Não dominância \\
\hline Idosos & 71,02 * & 89,56 * & 92,36 * & $-0,048$ & 0,000 & Cruzamento \\
\hline Idosos com diabetes & 78,10 * & 93,40 & 94,01 & $-0,026$ & 0,000 & Cruzamento \\
\hline Idosos com hipertensão & 70,74 * & 91,84 * & 93,31 * & $-0,047$ & 0,000 & Cruzamento \\
\hline
\end{tabular}

IUP: intersection union principle.

Fonte: Pesquisa Equidade na Saúde: Estratégia de Saúde da Família em Minas Gerais (2012).

* Diferença de médias em relação à media geral do grupo-alvo significativa a $1 \%$;

** Diferença de médias em relação à media geral do grupo-alvo significativa a $5 \%$. 
Figura 2

Curvas de concentração para visitas dos profissionais de saúde da Estratégia Saúde da Família nos 12 meses anteriores a data da entrevista em domicílios, segundo a presença de grupo alvo no domicílio. Minas Gerais, Brasil, 2012.

2a) Mulheres

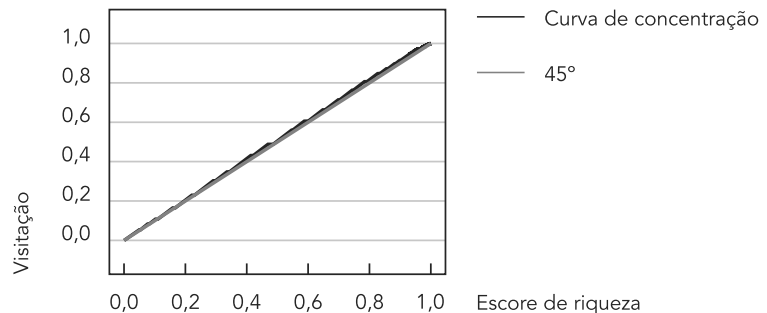

2c) Mulheres com hipertensão

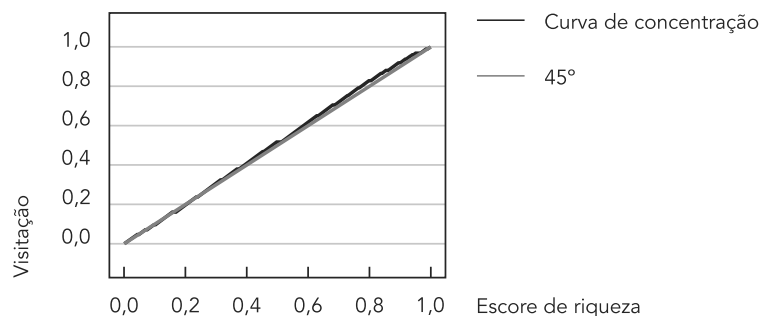

2e) Idosos com diabetes

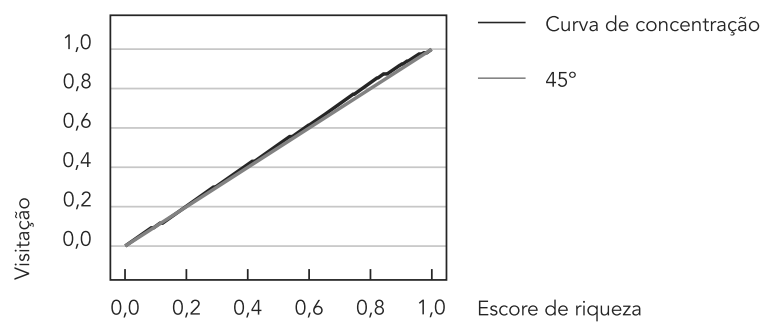

2g) Crianças

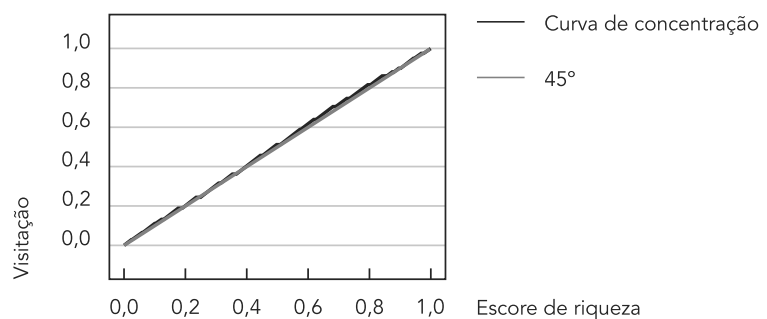

2b) Mulheres com diabetes

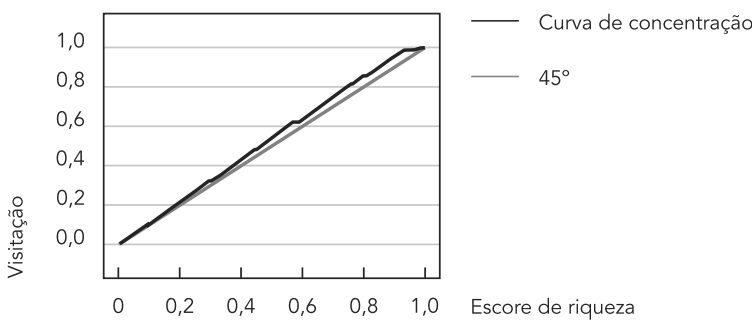

2d) Idosos

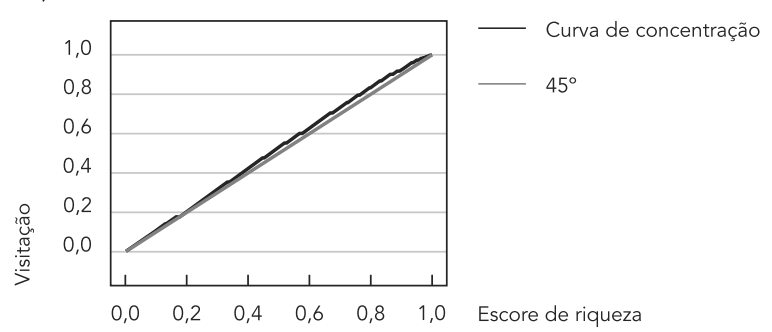

2f) Idosos com hipertensão

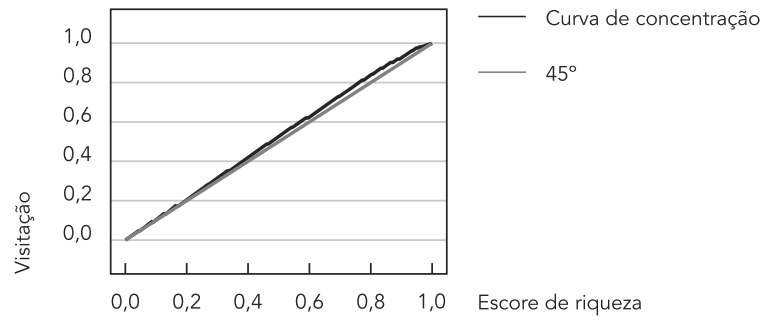

2h) Crianças com doenças crônicas

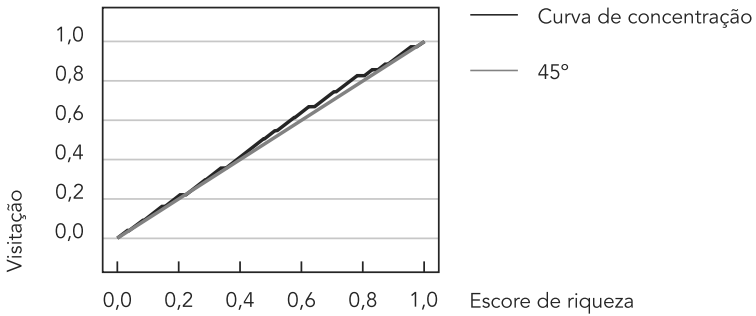

Fonte: Pesquisa Equidade na Saúde: Estratégia Saúde da Família em Minas Gerais (2012).

para todo o período analisado. Belo Horizonte está entre as capitais de maior cobertura populacional, com uma abrangência superior a $81,51 \%$ em setembro de 2014.
No contexto brasileiro de profundas desigualdades, a universalização da ESF pode ser uma política com impacto distributivo importante considerando, sobretudo, os mecanismos 
diretos e indiretos presentes na associação entre renda e saúde 24,25 . Inúmeros estudos são dedicados a avaliar os efeitos da ESF sobre a utilização dos serviços de saúde e sobre alguns marcadores de saúde. Foram observados efeitos positivos e significativos da ESF na redução da mortalidade, principalmente a infantil 26,27,28, e hospitalizações evitáveis por cuidado ambulatorial 29. Além disso, a ESF tem propiciado uma melhora no acesso a outros serviços, tais como, consultas médicas e odontológicas, e medicamentos necessários 19.

Apesar das dificuldades inerentes aos estudos avaliativos em saúde, as constatações do presente trabalho fornecem evidências de que a ESF tem se mostrado capaz de minimizar diferenças de acesso que há muito tempo se perpetuam. O rompimento com o padrão até então vigente é um importante desfecho obtido no Estado de Minas Gerais e fornece evidências que sustentam a opção pelo investimento no modelo de cuidados primários. Os achados destacam um padrão de equidade que favoreceu as famílias com menor perfil de renda, uma vez que evidencia a capacidade de ofertar serviços de saúde junto àqueles que mais necessitam. A escolha aleatória de domicílios deixa as evidências levantadas mais robustas e acentuam a sua importância.

Apesar dos avanços em termos da expansão da cobertura, Lima-Costa et al. 30 afirmam que a ESF ainda enfrenta múltiplos desafios. A importância de se abordar tais desafios, que ainda se colocam para a ESF, perpassa as ações para se garantir o acesso a serviços de saúde de qualidade. Alguns desses obstáculos apresentam-se como mais significativos e exigem ações corretivas em caráter de urgência, para que o futuro da ESF seja fortalecido. Dentre esses, pode-se destacar o modelo de atenção adotado no país. O Brasil é marcado por uma tripla carga de doenças (doenças agudas, crônicas e causas externas), com predomínio de doenças crônicas 31 . Apesar da adoção da ESF e da predominância das doenças crônicas no quadro sanitário do país, o modelo de sistema de saúde vigente ainda é voltado para a atenção a condições agudas 31 . Sabidamente, o modelo direcionado para intervenção nas condições agudas não é capaz de sustentar resultados sanitários satisfatórios quando tem de lidar com condições crônicas 32 . Outro tópico relevante que se coloca para os gestores da atenção primária brasileira perpassa a questão da gestão de recursos humanos (GRH). A saúde, especificamente, não acompanhou as evoluções observadas junto às organizações industriais, no que tange à importância da incorporação de novas práticas de GRH. Diante de uma situação marcada pela heterogeneidade e precariedade de vínculos de trabalho, pela necessidade de reformulação do processo de formação e capacitação, pela existência de um cenário de reorganização do modelo de saúde e pelo imperativo de atrair e fixar profissionais em áreas carentes, esse elemento emerge como um aspecto relevante a ser abordado para se efetivar a ESF de qualidade.

A superação desses desafios colocados tem a potencialidade de minimizar iniquidades na oferta de serviços de saúde. O processo de transição demográfica que a realidade brasileira vem enfrentando contribuirá para o aumento da prevalência de condições crônicas. Os dados obtidos aqui junto à realidade de Minas Gerais deixam claro que a ESF pode ser efetiva na abordagem dos problemas de saúde decorrentes de complicações inerentes a essas condições. Além disso, a confirmação da realização do acompanhamento satisfatório de famílias aleatoriamente escolhidas enfatiza a capilaridade dos programas com foco na atenção materno-infantil, doenças crônicas e de atenção a idosos.

A equidade é um dos princípios fundamentais do SUS e a ESF se mostrou como uma via capaz de garantir a materialização deste pilar. Para que a oferta de serviços atinja a todos aqueles que mais necessitam de cuidados é fundamental a boa execução do processo de trabalho das equipes de saúde. A carência de indícios, capazes de pontuar como as ações das equipes de saúde repercutem junto aos dados de saúde populacional, foi parcialmente abordada pelo presente trabalho. O desenvolvimento de políticas públicas que fomentem a expansão da ESF, garantindo a qualidade e o cumprimento adequado do processo de trabalho em saúde, pode potencializar resultados em saúde satisfatórios, no que tange à melhora de indicadores de saúde populacional.

A principal limitação da pesquisa diz respeito à validade externa dos resultados obtidos para o Estado de Minas Gerais. O estudo tem ampla validade interna, uma vez que a amostra é representativa para a área urbana de Minas Gerais e para os grupos-alvo investigados. A despeito do fato de a ESF ter diretrizes nacionais definidas no escopo do SUS, há diferenças na implementação e gestão destes programas entre as Unidades da Federação e entre os municípios. Embora se reconheça essa limitação, a pesquisa se concretiza como um estudo de caso importante com evidências claras do papel da ESF como provedor da atenção primária equitativa, principalmente, para as camadas de renda mais baixas. 


\section{Resumen}

La Estrategia de Salud Familiar (ESF) tiene un papel importante en la prevención y el seguimiento de las familias en el Sistema Único de Salud. Este estudio analiza la equidad en la cobertura de estos servicios ofertados en el área urbana de Minas Gerais, Brasil. La investigación es inédita y analiza marcadores para cuatro grupos objetivo: mujeres, mujeres embarazadas, niños y ancianos. La muestra es representativa por macrorregiones de salud. En 2012, fueron investigados 6.797 domicilios y fueron entrevistados 5.820 mujeres, 1.758 niños y 3.629 adultos mayores. Para el análisis de la equidad, se construyeron las tasas de cobertura por clase de riqueza y se estimaran los índices y curvas de concentración. Los resultados revelan que el ESF es una política equitativa. Los indicadores muestran que los hogares más pobres tienen mayores tasas de visitas de ESF. Teniendo en cuenta la población elegible, la cobertura es muy alta: el 88\% de la población recibió al menos una visita de profesionales de la ESF en los últimos 12 meses, lo que resulta en índices de concentración cerca de cero.

Estrategia de Salud Familiar; Atención Primaria de Salud; Equidad en Salud

\section{Colaboradores}

M. V. Andrade participou de todas as etapas do trabalho. K. Noronha participou da concepção do desenho do estudo e do desenho amostral, construção e análise do banco de dados, definição dos indicadores, estimação das curvas e índices de concentração, análise estatística e redação do artigo. A. C. Q. Barbosa participou da concepção do desenho de estudo e desenho amostral, análise dos resultados, construção dos indicadores e leitura crítica da versão final do artigo. T. A. H. Rocha participou da concepção do desenho de estudo e do desenho amostral, pesquisa de campo e discussão dos resultados, elaboração da máscara do software eletrônico da pesquisa e leitura crítica da versão final do artigo. N. C. Silva participou da concepção do desenho de estudo e do desenho amostral, pesquisa de campo e leitura crítica da versão final do artigo. J. A. Calazans participou da concepção do desenho de estudo e do desenho amostral, pesquisa de campo, discussão dos resultados, organização do banco de dados, análise estatística descritiva e redação final. M. N. Souza participou da organização do banco de dados, análise estatística descritiva, discussão dos resultados, construção de indicadores, estimação das curvas e índices de concentração e elaboração da versão final do texto. L. R. Carvalho participou do georreferenciamento das unidades básicas de saúde, participou do piloto amostral da pesquisa, organização do banco de dados, análise estatística descritiva, discussão dos resultados, construção de indicadores, estimação das curvas e índices de concentração e leitura crítica da versão final do artigo. A. Souza participou da organização do banco de dados, análise estatística descritiva, discussão dos resultados, construção de indicadores, estimação das curvas e índices de concentração e elaboração da versão final do texto, incluindo a formatação.

\section{Agradecimentos}

Esta pesquisa foi financiada pela Secretaria de Estado de Saúde de Minas Gerais (SES-MG). Os autores agradecem os comentários dos técnicos da SES-MG que participaram de seminários prévios à versão final deste trabalho. Agradecimento especial a Eugênio Vilaça, que participou indiretamente de todas as etapas do trabalho, opinando e compartilhando a sua vasta experiência sobre o SUS, em particular no Estado de Minas Gerais. 


\section{Referências}

1. Victora C, Barreto ML, Leal MC, Monteiro CA, Schmidt MI, Paim J, et al. Health conditions and health-policy innovations in Brazil: the way forward. Lancet 2011; 377:2042-53.

2. Paim JS, Travassos CMR, Almeida CM, Bahia L, Macinko J. The Brazilian health system: history, advances, and challenges. Lancet 2011; 377:1778-97.

3. Medici A. Proposta para melhorar a cobertura, a eficiência e a qualidade no setor saúde. In: Bacha EL, Schwartzman S, organizadores. Brasil: a nova agenda social. Rio de Janeiro: Instituto de Estudos de Política Econômica - Casa das Garças; 2011. p. 23-93.

4. Andrade MV, Noronha K, Barbosa AC, Silva NC, Rocha TAH, Arantes RC, et al. Equidade na saúde: o Programa de Saúde da Família em Minas Gerais. Belo Horizonte: Autêntica Editora; 2013.

5. Porto SM, Ugá MAD, Moreira RS. Uma análise da utilização de serviços de saúde por sistema de financiamento: Brasil 1998-2008. Ciênc Saúde Coletiva $2011 ; 16: 3795-806$.

6. Fuhrmann NL. Programa Saúde da Família: viabilizando a saúde pública universalizada no Brasil. Textos contextos (Porto Alegre) 2003; 2(1). http:// revistaseletronicas.pucrs.br/fo/ojs/index.php/ fass/article/view/966.

7. Lentsck MH, Kluthcovsky ACGC, Kluthcovsky FA. Avaliação do Programa Saúde da Família: uma revisão. Ciênc Saúde Coletiva 2010; 15:3455-66.

8. Ministério da Saúde. Política Nacional de Atenção Básica. Brasília: Ministério da Saúde; 2006.

9. Ministério da Saúde. Portaria no 2.488 de 21 de outubro de 2011. Diário Oficial da União 2011; 21 out.

10. Corbo ADA, Morosini MVGC. Saúde da família: história recente da reorganização da atenção à saúde. In: Escola Politécnica de Saúde Joaquim Venâncio, organizador. Textos de apoio em políticas de saúde. Rio de Janeiro: Editora Fiocruz; 2005. p. 157-81.

11. Travassos C, Viacava F, Fernandes C, Almeida CM. Desigualdades geográficas e sociais na utilização de serviços de saúde no Brasil. Ciênc Saúde Coletiva $2000 ; 5: 133-49$.

12. Nunes A, Santos JRS, Barata RB, Vianna SM. Medindo as desigualdades em saúde no Brasil: uma proposta de monitoramento. Brasília: Organização Pan-Americana da Saúde/Instituto de Pesquisa Econômica Aplicada; 2001.

13. Neri M, Soares W. Desigualdade social e saúde no Brasil. Cad Saúde Pública 2002; 18:77-87.

14. Travassos C, Oliveira EXG, Viacava F. Desigualdades geográficas e sociais no acesso aos serviços de saúde no Brasil: 1998 e 2003. Ciênc Saúde Coletiva 2006; 11:975-86

15. Campino ACC, Diaz MDM, Paulani LM, Piola S, Oliveira RG. Poverty and equity in health in Latin America and Caribbean: results of country-case studies from Brazil, Ecuador, Guatemala, Jamaica, Mexico and Peru. Washington DC: The World Bank/United Nations Development Program/Pan American Health Organization; 1999.
16. Almeida C, Travassos C, Porto S, Labra ME. Health sector reform in Brazil: a case study of inequity. Int J Health Serv 2000; 30:129-62.

17. Viacava F, Travassos C, Pinheiro R, Brito A. Gênero e utilização de serviços de saúde no Brasil. Washington DC: Organização Pan-Americana da Saúde; 2001.

18. Szwarcwald CL, Souza-Júnior PR, Damacena GN. Socioeconomic inequalities in the use of outpatient services in Brazil according to health care need: evidence from the World Health Survey. BMC Health Serv Res 2010; 10:1-7.

19. Macinko J, Lima-Costa MF. Access to, use of and satisfaction with health services among adults enrolled in Brazil's Family Health Strategy: evidence from the 2008 National Household Survey. Trop Med Int Health 2012; 17:36-42.

20. Souza MF. O Programa Saúde da Família no Brasil: análise do acesso à atenção básica. Rev Bras Enferm 2008; 61:153-8.

21. Assis MMA, Cerqueira EM, Jesus WLA, Nascimento MAA, Santos AM. Atenção primária à saúde e sua articulação com a Estratégia Saúde da Família: construção política, metodológica e prática. Rev APS 2007; 10:189-99.

22. Secretaria de Estado de Saúde. Deliberação CIBSUS/MG no 978, de 16 de novembro de 2011. Diário Oficial de Minas Gerais 2011; 16 nov.

23. O'Donnell O, van Doorslaer E, Wagstaff A, Lindelow M. Analyzing health equity using household survey data. Washington DC: The World Bank; 2008.

24. Tomasi E, Facchini LA, Thumé E, Piccini RX, Silveira O, Siqueira FV, et al. Características da utilização de serviços de Atenção Básica à Saúde nas regiões Sul e Nordeste do Brasil: diferenças por modelo de atenção. Ciênc Saúde Coletiva 2011; 16:4395-404.

25. Facchini LA, Piccini RX, Tomasi E, Thumé E, Silveira D, Siqueira FCV, et al. Desempenho do PSF no Sul e no Nordeste do Brasil: avaliação institucional e epidemiológica da atenção básica à saúde. Ciênc Saúde Coletiva 2006; 11:669-81.

26. Macinko J, Guanais FC, Souza MFM. An evaluation of the impact of the Family Health Program on infant mortality in Brazil, 1990-2002. J Epidemiol Community Health 2006; 60:13-9.

27. Rocha R, Soares R. Impacto de programas de saúde a nível familiar e comunitário: evidências do Programa Saúde da Família. In: Encontro Nacional da ANPEC. http://www.anpec.org.br/encon tro2008/artigos/200807211610510-.pdf (acessado em 15/Ago/2013).

28. Mendonça CS, Harzheim E, Duncan BB, Nunes LN, Leiyh W. Trends in hospitalizations for primary care sensitive conditions following the implementation of Family Health Teams in Belo Horizonte, Brazil. Health Policy Plan 2012; 27:348-55.

29. Macinko J, Dourado I, Aquino R, Bonolo PF, LimaCosta MF, Medina MG, et al. Major expansion of Primary Care in Brazil linked to decline in unnecessary hospitalization. Health Aff 2010; 29:2149-60. 
30. Lima-Costa MF, Turci MA, Macinko J. Estratégia Saúde da Família em comparação a outras fontes de atenção: indicadores de uso e qualidade dos serviços de saúde em Belo Horizonte, Minas Gerais, Brasil. Cad Saúde Pública 2013; 29:1370-80.

31. Mendes EV. As redes de atenção à saúde. Brasília: Organização Pan-Americana da Saúde; 2011.
32. Organização Mundial da Saúde. Cuidados inovadores para condições crônicas: componentes estruturais de ação. Brasília: Organização Mundial da Saúde; 2003.

Recebido em 27/Ago/2014

Versão final reapresentada em 08/Dez/2014

Aprovado em 06/Jan/2015 